\title{
The Influence of Different Exercise Intervention Programs on Changes in Quality of Life and Activity of Daily Living Levels among Geriatric Nursing Home Residents
}

\author{
Ryo TAKeUchi, RPT, MEd ${ }^{1,3)}$, Yoshiro Hatano, PhD'2), MASAHIRO YAMASAKI, PhD ${ }^{3)}$ \\ ${ }^{1)}$ Shizuoka Health Institute, 2276 Yata, Mishima, Shizuoka 411-0801, Japan. \\ TEL: +81 55-973-7001, FAX: +81 55-973-7010,E-mail: ryotakeuchi@tiger.odn.ne.jp \\ ${ }^{2)}$ Tokyo Gakugei University School of Education \\ ${ }^{3)}$ Hiroshima University Graduate School of Integrated Arts and Science
}

\begin{abstract}
Purpose] The purpose of this study was to examine the effects of group-exercise and individualized exercise programs on factors associated with physical function, activities of daily living (ADL), and quality of life (QOL) among nursing home residents. [Subjects and Methods] This study examined differences in changes of physical function, ADL, and QOL levels among participants undergoing 2 exercise intervention programs. The participants were drawn from a geriatric nursing home population and were randomly divided into 2 groups: group exercise intervention $(\mathrm{n}=14$; mean age, $85.7 \pm 3.9$ years $)$ and individual exercise intervention $(\mathrm{n}=12$; mean age, $85.8 \pm 7.8$ years). Group therapy consisted of individualized rehabilitation along with a group outdoor walking program; individual therapy consisted of individualized rehabilitation alone. Physical function, ADL, and QOL levels were measured 4 times (approximately once per month). [Results] For both measures of QOL, an interaction was seen between the group exercise intervention group and the individual exercise intervention group. No interactions were seen between groups for physical function or ADL. [Conclusion] In designing a physical exercise program for residents in geriatric nursing home, it is important to conduct part of the program outside and to include social activity elements.
\end{abstract}

Key words: Quality of life, Group exercise, Geriatric nursing home residents

(This article was submitted Jun. 28, 2010, and was accepted Aug. 24, 2010)

\section{INTRODUCTION}

Japan has become the world's leading country in terms of longevity ${ }^{1)}$. In 2006, the Japanese public long-term care insurance system was revised to address the increase in the number of elderly people requiring nursing care as well as the level of nursing care needed ${ }^{2}$. This revision includes the concept of placing the same importance on disease severity as is used in hospitals when providing physical therapy services in geriatric nursing homes ${ }^{2}$, and it may indicate that a pervasive effect of physical therapy is expected. It is known that physical function and the performance of activities of daily living (ADL) can be improved using physical therapy services ${ }^{3-7)}$, and providing individualized exercise programs may allow elderly people to continue performing ADL independently for longer periods. Residents at geriatric homes likely have lower selfassessments of their quality of life (QOL) based on the stress of being in a nursing home ${ }^{8)}$. Improving living environment and lifestyle is one way of improving residents' $\mathrm{QOL}^{8)}$, and it is thought that such improvements should focus on the performance of ADL as well as social aspects of life.
Previous studies on the effect of exercise intervention programs for elderly residents of nursing homes have primarily focused on physical function and ADL, with few studies examining social aspects. Ruuskanen et al. $\left.{ }^{9}\right)$ reported improvements in QOL among nursing home residents who participated in a walking program along with indoor gymnastics. In contrast, McRae et al. ${ }^{7)}$ reported no changes in QOL when residents undertook a walking program. However, those studies used different forms of physical exercise, which could account for their different results. To determine the most effective exercise for nursing home residents, with a focus on QOL, studies need to compare the effects of different exercise intervention programs on QOL.

Geriatric nursing homes in Japan are generally thought of as facilities that help residents to later return home; thus, physical therapy is their main service, and all residents undergo physical therapy programs. The purpose of this study was to examine the effects of a group exercise program and an individual exercise program on factors associated with physical strength, ADL, and QOL among nursing home residents. 


\section{SUBJECTS AND METHODS}

The 28 study participants ( 4 men and 24 women) were drawn from among the population of a geriatric nursing home (maximum capacity of 134) using the following inclusion criteria: a cognitive function score of 21 points or higher as assessed by the Revised Hasegawa's Dementia Scale ${ }^{10)}$, and the ability to walk continuously for more than 20 min. Participants were randomly divided into two groups: the group exercise intervention $(\mathrm{n}=14$; mean age, $85.7 \pm 3.9$ years, Body Mass Index, $21.0 \pm 2.9)$, and the individual exercise intervention $(\mathrm{n}=14$, mean age, $85.8 \pm 7.8$ years, Body Mass Index, $20.6 \pm 3.5)$. However, two participants in the individual exercise group withdrew from the intervention, so only the results of the remaining 12 were analyzed. The group exercise intervention consisted of individualized physical therapy along with a group outdoor walking program; the individual exercise intervention consisted of individualized physical therapy alone.

Written informed consent was obtained from all the study participants. The facility manager and head of each ward conferred at a committee and approved the study. Regarding the anonymity of the questionnaires given to the participants, they and the nursing home administration were informed of our intentions to use only numerical values when reporting the results.

Physical function was assessed by gait velocity $(\mathrm{m} / \mathrm{min})$, step length $(\mathrm{cm})$, and standing on one leg with eyes open (sec). Each participant walked at his/her normal pace along a 14-m straight line, and gait velocity was calculated as the time needed to pass from $2 \mathrm{~m}$ to $12 \mathrm{~m}$. Step length was obtained by measuring the stride length at around the halfway point of the 14-m line and dividing by 2 . Standing on one legged with eyes open was measured by having the participant place hands on the waist while standing on one leg. The length of time was measured from the time the foot was raised until it was placed back on the ground. This time was recorded for each leg, and the longer time was used. However, if the standing leg moved or hands were removed from the waist, the measurement was suspended.

ADL was assessed using the Barthel Index ${ }^{11)}$. This index measures the following variables: ability to eat certain foods, moving from a wheelchair to a bed and returning, personal hygiene (washing face, combing hair, shaving, brushing teeth), getting on and off the toilet (handling clothes, wiping, flushing), bathing, walking on a level surface (or if unable to walk, propelling a wheelchair), ascending and descending stairs, dressing (including tying shoes, using fasteners), bowel, control and bladder control. The overall score ranges from 0 to 100 . QOL was assessed using the Life Satisfaction Index A (LSIA) ${ }^{12-14)}$ and the Philadelphia Geriatric Center Morale Scale (PGC) $)^{15-17)}$.

Physical function, ADL, and QOL of all participants were assessed once per month for 4 months (Evaluations 1 to 4 ).

Between Evaluation 1 and Evaluation 2, all participants underwent individual physical therapy twice per week for 20 min. Individual physical therapy consisted of resistance training for the lower extremities, balance exercises, and indoor gait exercises; the choice of therapy was based on each participant's needs. Exercise intensity was determined based on a Ratings of Perceived Exertion scale (6 to 20, not exceeding 11 [fairly light] ${ }^{18)}$. After Evaluation 2, individual physical therapy was conducted once a week for the group therapy exercise group; an additional exercise intervention was also added that consisted of a 20-min group walk around the facility's walking trail. Each group walk comprised 4 to 5 participants and a physical therapist; the walking program was recorded by a surveillance system for security purposes. In case of bad weather, the outdoor walking program was rescheduled for the preceding or following day. Participants in the individual exercise intervention group continued to receive individual physical therapy twice per week. Thus, both groups underwent a total of 8 exercise interventions between evaluations. Whenever someone was not able to participate in the intervention due to poor health, that intervention was rescheduled for the following week.

Monthly changes in physical function, ADL, and QOL of both groups were assessed through two-way analysis of variance, and the interaction of change was clarified in both recordings. When an interaction was identified, multiple comparisons were carried out using the Wilcoxon signed rank test among the four evaluation sessions, and the MannWhitney test between the two intervention groups. The significance level was chosen as $<0.05$. SPSS version 16 was used for all analyses (SPSS Japan Inc., Tokyo, Japan).

\section{RESULTS}

For LSIA, an interaction was seen between the group exercise intervention group and the individual exercise intervention group $(\mathrm{F}=3.761, \mathrm{p}<0.05$; Table 1$)$. As a result of multiple comparisons, LSIA scores changed significantly among the four evaluation periods in the group exercise intervention group. However, no significant differences were recognized between the group exercise intervention group and the individual exercise intervention group.

For PGC, an interaction was also seen between the group exercise intervention group and the individual exercise intervention group $(\mathrm{F}=5.562, \mathrm{p}<0.01$; Table 1$)$. As a result of multiple comparisons, PGC scores changed significantly among the four evaluation periods in the group exercise intervention group. However, no significant differences were recognized between the group exercise intervention group and the individual exercise intervention group.

As measured with the Barthel Index, most participants had restrictions in their ability to climb up and down stairs. Independence was maintained at a high level, although some participants needed assistance for bathing and grooming. Neither an interaction nor a main effect for ADL was recognized between the group exercise intervention group and the individual exercise intervention group (Table 1).

No significant differences were recognized in either interaction or main effect between the group exercise intervention group and the individual exercise intervention group in gait velocity, step length, or one-legged standing with eyes open (Table 1). 
Table 1. Differences in changes in scores between Group-exercise and Individual groups

\begin{tabular}{lccccc}
\hline & & Evaluation 1 & Evaluation 2 & Evaluation 3 & Evaluation 4 \\
\hline LSIA(0-20) & Group-exercise & $7.9 \pm 3.4$ & $8.1 \pm 2.9$ & $10.3 \pm 2.4$ & $10.6 \pm 3.2$ \\
& Individual & $8.6 \pm 3.0$ & $8.7 \pm 3.0$ & $8.7 \pm 2.8$ & $8.7 \pm 3.7$ \\
& & & $\mathrm{~F}=3.761 \mathrm{p}<0.05$ \\
\hline PGC(0-11) & Group-exercise & $6.3 \pm 2.7$ & $5.4 \pm 2.6$ & $7.5 \pm 2.9$ & $7.2 \pm 3.1$ \\
& Individual & $6.4 \pm 2.0$ & $7.1 \pm 1.6$ & $6.6 \pm 2.3$ & $6.9 \pm 2.1$ \\
& & & $\mathrm{~F}=5.562 \mathrm{p}<0.01$ \\
\hline Gait velocity & Group-exercise & $39.7 \pm 9.3$ & $40.4 \pm 8.1$ & $46.2 \pm 11.1$ & $43.4 \pm 10.1$ \\
(m/min) & Individual & $40.5 \pm 12.2$ & $39.6 \pm 11.5$ & $38.6 \pm 11.0$ & $40.3 \pm 12.6$ \\
& & & $\mathrm{~F}=2.796$ Not significant \\
\hline Step length & Group-exercise & $42.2 \pm 8.7$ & $43.7 \pm 8.2$ & $44.9 \pm 8.9$ & $44.8 \pm 8.4$ \\
(cm) & Individual & $37.0 \pm 8.4$ & $36.2 \pm 8.4$ & $37.5 \pm 8.2$ & $35.1 \pm 7.4$ \\
& & & $\mathrm{~F}=1.260 \mathrm{Not}$ significant \\
\hline One-legged standing & Group-exercise & $2.6 \pm 2.6$ & $2.8 \pm 2.3$ & $2.4 \pm 2.1$ & $2.7 \pm 2.6$ \\
with eyes open (sec) & Individual & $4.7 \pm 6.3$ & $5.3 \pm 7.4$ & $5.5 \pm 5.8$ & $6.1 \pm 7.7$ \\
& & & $\mathrm{~F}=0.603$. Not significant \\
\hline Barthel Index & Group-exercise & $89.6 \pm 9.1$ & $89.6 \pm 8.4$ & $90.0 \pm 7.8$ & $90.0 \pm 7.8$ \\
(0-100) & Individual & $86.3 \pm 6.1$ & $85.8 \pm 6.0$ & $85.4 \pm 5.8$ & $85.4 \pm 5.8$ \\
& & & & $\mathrm{~F}=1.318$ Not significant \\
\hline
\end{tabular}

Two-way layout analysis of variance; Group: $n=14$; Individual: $n=12$. Values are mean \pm SD. LSIA $=$ Life Satisfaction Index A; PGC $=$ Philadelphia Geriatric Center Morale Scale. LSIA: Group-exercise significant 1st to 3rd**, 1 st to 4 th**, 2nd to 3 rd**, 2nd to 4 th $^{* *}$. $* *$ : $\mathrm{p}<0.01$. PGC: Group-exercise significant 1 st to $3 \mathrm{rd}^{* *}, 1$ st to 4 th**, 2 nd to $3 \mathrm{rd}^{* *}$, 2nd to 4 th*.

$*: \mathrm{p}<0.05, * *: \mathrm{p}<0.01$

\section{DISCUSSION}

We assessed the effects of two different exercise intervention programs on QOL, ADL, and physical function among 26 nursing home residents in Japan. We decided to conduct the exercise intervention designed by Ano et al. ${ }^{19)}$, with a focus on QOL in this study; therefore we thought it practical to use the LSIA and PGC scales. The participants in this study were elderly residents, whereas those in the study by Ano et al. ${ }^{19)}$ were elderly inpatients; both studies included participants who were not living at home. LSIA, which was used in this study to assess QOL, was developed by Neugarten et al. ${ }^{14)}$ and has been used in the area of psychosociology that focuses on the elderly ${ }^{12-14)}$; we used a modified Japanese version of the scale ${ }^{12}$. PGC is an indicator created by Lawton ${ }^{15)}$ that measures subjective well-being; a short, modified, Japanese version was used in this study ${ }^{16,17)}$.

The Barthel Index is commonly used to assess ADL in the physical therapy field as well as in clinical practice because this index is both reliable and simple ${ }^{11,20)}$. In addition, because the Barthel Index can be used for longitudinal studies $^{20)}$, we thought it would be useful in the present study.

In this study, research was started 1 month prior to the initial intervention of the group outdoor walking program, so as to reduce possible confounding factors. The intervention of group outdoor walking occurred between Evaluation 1 and Evaluation 2. From Evaluation 2 to Evaluation 3, there was a change of 2.2 points in LSIA, whereas a change of only 0.1 to 0.3 points was seen at the other stages. Similarly, for the PGC, there was a change of
2.1 points from Evaluation 2 to Evaluation 3, whereas a change of only $0.3-0.9$ points was seen at other stages. For both the LSIA and PGC, the largest changes in scores were seen after the intervention method was altered in the group exercise intervention group. These results support those obtained by Ruuskanen et al. ${ }^{9)}$, that an intervention of walking combined with indoor exercise was associated with contentment with life among nursing home residents.

Takeuchi et al. ${ }^{21)}$ reported that QOL scales changed and that an outdoor walking program produced a sense of life satisfaction and subjective wellbeing among geriatric nursing home residents. In that study of QOL, the fact that there was improvement in such items as life satisfaction and subjective wellbeing for the combination group can be considered a sign that the outdoor walking program worked positively. The outdoor walking program used in our study was carried out in a group setting, and at a pace that allowed walkers to see the landscape and have social interactions with a walking companion. This highlights how social networks with companions along with recreation can improve subjective wellbeing and life satisfaction among elderly people ${ }^{22,23)}$. It is likely that this type of program serves as a functional training factor as well as recreation. Therefore, it was thought that the group exercise intervention led to the improvement of QOL among nursing home residents.

In terms of physical factors and monthly changes in ADL, it is likely that a certain intensity of physical exercise is needed for any improvement to be seen. Fiatarone et al. ${ }^{5)}$ reported that when a muscle strengthening exercise program was performed for geriatric nursing home residents three times a week for 10 weeks at an intensity of $80 \%$ of the 
maximum muscular force, muscular force increased 2.1fold, and gait velocity increased $12 \%$. Evans ${ }^{4,24)}$ reported that participants showed improvement in muscular force and physical function when muscle strength exercises were performed by geriatric nursing home residents at the intensity of $60 \%$ of the repetition maximum. In this study, both groups' exercise intensities corresponded to a score of about 11 (fairly light) on the Ratings of Perceived Exertion Scale ${ }^{18)}$. Therefore, this low level of exercise intensity might explain the lack of outcome in physical factors and ADL. Kubota et al. ${ }^{6}$ ) reported that gait velocity was improved in elderly residents who underwent either group or individual exercise interventions. However, in that study, participants walked for 2 hours each time, which is six times as long as the time used in this study. This difference may explain the lack of changes in physical factors seen in this study. However, considering the age of our participants, it is significant that physical function was not decreased in this study but instead was maintained.

In terms of limitations, this study was conducted in a single nursing home in Japan, and the number of participants was small. Therefore, this study may not be generalizable to a larger population. Although we had planned to use a crossover design following Evaluation 3 in this study, too few residents agreed to participate for the time needed for a crossover design. Therefore, the same program was continuously executed after Evaluations 3 and 4 for both groups. Thus, it is likely that this study does not have enough power to prove that QOL was improved by different exercise interventions.

\section{REFERENCES}

1) Health and Welfare Statistics Association: Annual Statistical Report of National Health Conditions. Tokyo, 2008, pp 236-239 (in Japanese).

2) Health and Welfare Statistics Association: Annual Statistical Report of National Health Conditions. Tokyo, 2008, pp 70-71 (in Japanese).

3) Baum EE, Jarjoura D, Polen AE, et al.: Effectiveness of a group exercise program in a long-term care facility: a randomized pilot trial. J Am Med Directors Assoc, 2003, 42: 74-80.

4) Evans WJ: Effect of exercise on body composition and functional capacity of the elderly. J Gerontol A Biol Sci Med Sci, 1995, 50: 147-150.
5) Fiatarone MA, O'Neill EF, Ryan ND, et al.: Exercise training and nutritional supplementation for physical frailty in very elderly people. N Engl J Med, 1994, 330: 1769-1775.

6) Kubota A, Hatano Y: Effect of positive exercise on quality of life. Progress in Soc Welfare Res, 2006, 1: 61-69 (in Japanese).

7) McRae PG, Asplund LA, Schnelle JF, et al.: A walking program for nursing home residents: effects on walk endurance, physical activity, mobility, and quality of life. J Am Geriatr Soc, 1996, 44: 175-180.

8) Iriuchijima K, Aoki K: The relationship between the environmental factor recognizing stresses in the living environment and quality of life in geriatric facilities. J Tokyo Academy Health Sci, 2002, 5: 75-85 (in Japanese).

9) Ruuskanen JM, Parkatti T: Physical activity and related factors among nursing home residents. J Am Geriatr Soc, 1994, 42: 987-991.

10) Imai $Y$, Hasegawa K: The Revised Hasegawa's Dementia Scale (HDS-R) Evaluation of its usefulness as a screening test for dementia. J Hong Kong Coll. Psychiatr, 1994, 4(SP2): 20-24.

11) Mahoney FI, Barthel DW: Functional evaluation: The Barthel Index. Md State Med J, 1965, 14: 61-65.

12) Koyano PW: Common dimensions and interrelationships of the measure of morale, life satisfaction and happiness of the aged: A re-examination. Jpn J Gerontol, 1983, 5: 129-142 (in Japanese).

13) Liang J: Dimensions of the Life satisfaction Index A: structural formulation. J Gerontol, 1984, 39: 613-622.

14) Neugarten BL, Havighurst RJ, Tobin SS: The measurement of life satisfaction. J Gerontol, 1961, 6:134-143.

15) Lawton MP: The Philadelphia Geriatric Center Morale Scale: a revision. J Gerontol, 1975, 30: 85-89.

16) Liang J, Asano H, Bollen KA, et al.: Cross-cultural comparability of the Philadelphia Geriatric Center Morale Scale: an American-Japanese comparison. J Gerontol, 1987, 42: 37-43.

17) Koyano PW: An analysis of the revised Philadelphia Geriatric Center morale scale. Jpn J Gerontol, 1981, 3: 83-95 (in Japanese).

18) Noble BJ, Borg GAV, Jacobs I, et al.: A category-ratio perceived exertion scale. Med Sci Sports Exerc, 1983, 15: 523-528.

19) Ano $Y$, Higashi T, Okita M, et al.: QOL for elderly inpatients compared with outpatients who receive day-care service. Occup Ther, 1998, 17: 273-279 (in Japanese).

20) Nagano K: Appropriate outcome measure for evaluating change in activities of daily living of elderly residents. Jpn J Public Health, 2002, 49: 76-87 (in Japanese).

21) Takeuchi R, Hatano Y: The effect of group outdoor walking intervention upon changes in the profile of mood status, ADL and QOL -Among the geriatric health nursing care-house participants-. Progress Soc Welfare Res, 2008, 3: $32-41$ (in Japanese).

22) Arling, G: The elderly widow and her family, neighbors and friends. J Marriage and the Family, 1976, 38: 757-768.

23) Brown BA, Frankel BG: Activity through the years: Leisure, Leisure satisfaction, and life satisfaction. Sociology of Sport J, 1993, 10: 1-17.

24) Evans WJ: Exercise training guidelines for the elderly. Med Sci Sports Exerc, 1999, 31: 12-17. 